\title{
Initial results of a direct comparison between the Surface Detectors of the Pierre Auger Observatory and the Telescope Array
}

R. Takeishi ${ }^{*}, 1$, , R. Cady $^{b}$, J. N. Matthews ${ }^{b}$, T. Nonaka ${ }^{a}$, S. Ogio ${ }^{c}$, H. Sagawa ${ }^{a}$ for the Telescope Array Collaboration ${ }^{\dagger}$, C. Covault ${ }^{d}$, T. Fujii $^{e}$, J. Johnsen $^{f}$, P. Lebrun $^{g}, \mathbf{R}$. Lorek $^{d}$, P. Mantsch ${ }^{g}$, J. A. J. Matthews ${ }^{h}$, P. Mazur ${ }^{g}$, S.Quinn ${ }^{d}$, F. Sarazin ${ }^{f}$, R. Sato ${ }^{i}$, for the Pierre Auger Collaboration ${ }^{\ddagger}$, S. Collonges ${ }^{j}$, B. Courty ${ }^{j}$, B. Genolini ${ }^{k}$, L.

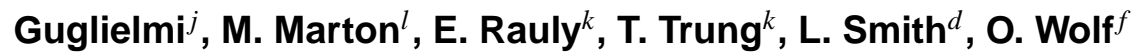

${ }^{a}$ Institute for Cosmic Ray Research, University of Tokyo, Kashiwa, Chiba, Japan

${ }^{b}$ High Energy Astrophysics Institute and Department of Physics and Astronomy, University of Utah, Salt Lake City, UT, USA

${ }^{c}$ Graduate School of Science, Osaka City University, Osaka, Osaka, Japan

${ }^{d}$ Department of Physics, Case Western Reserve University, Cleveland OH, USA

${ }^{e}$ University of Chicago, Enrico Fermi Institute, Chicago IL, USA

${ }^{f}$ Physics Department, Colorado School of Mines, Golden CO, USA

${ }^{g}$ Fermi National Laboratory, Batavia IL, USA

${ }^{h}$ Department of Physics and Astronomy, University of New Mexico, Albuquerque, NM, USA

${ }^{i}$ Pierre Auger Observatory, Malargüe, Argentina

${ }^{j}$ Laboratoire Astroparticules et Cosmologie (APC), Université Paris 7, CNRS-IN2P3, Paris, France

${ }^{k}$ Institut de Physique Nucléaire d'Orsay (IPNO), Université Paris 11, CNRS-IN2P3, Orsay, France

${ }^{l}$ Laboratoire de Physique Subatomique et de Cosmologie (LPSC), Université Joseph Fourier, INPG, CNRS-IN2P3, Grenoble, France

${ }^{1}$ E-mail: takedicrr.u-tokyo.ac.jp 
The Pierre Auger Observatory (Auger) in Mendoza, Argentina and the Telescope Array (TA) in Utah, USA aim at unraveling the origin and nature of Ultra-High Energy Cosmic Rays (UHECR). At present, there appear to be subtle differences between Auger and TA results and interpretations. Joint working groups have been established and have already reported preliminary findings. From an experimental standpoint, the Surface Detectors (SD) of both experiments make use of different detection processes not equally sensitive to the components of the extensive air showers making it to the ground. In particular, the muonic component of the shower measured at ground level can be traced back to the primary composition, which is critical for understanding the origin of UHECRs. In order to make direct comparisons between the SD detection techniques used by Auger and TA, a joint SD experimental research program is being developed. In the first phase, two Auger SD stations were deployed at the TA Central Laser Facility to compare station-level responses. This paper concentrates on the results obtained with the first Auger SD station (an "Auger North" design), which has been operating since October 2014. The second Auger SD station, identical to the ones being operated at Auger in Argentina (an "Auger South" design), was just deployed in June 2015. The second phase of this research program will be to co-locate six Auger North SD stations with TA stations in the field to compare event-level responses.

The 34th International Cosmic Ray Conference,

30 July- 6 August, 2015

The Hague, The Netherlands

\footnotetext{
*Speaker.

${ }^{\dagger}$ For full author list and acknowledgments see http://www.telescopearray.org/images/papers/ICRC2015-authorlist.pdf

${ }^{\ddagger}$ For full author list and acknowledgments see http://www.auger.org/archive/authors_2015_ICRC.html
} 


\section{Introduction}

The Pierre Auger Observatory [1], which is located in Mendoza province, Argentina, is a hybrid instrument to detect cosmic-ray induced air showers. It combines a surface detector array (SD) with fluorescence telescopes making up the fluorescence detector (FD) overlooking the SD. The Auger SD consists of 1660 water-Cherenkov stations placed on a triangular grid with $1.5 \mathrm{~km}$ spacing, covering an area of $3000 \mathrm{~km}^{2}$. The Telescope Array (TA) experiment [2], which is located in western Utah, USA, consists of 507 scintillation counters, placed on a square grid with $1.2 \mathrm{~km}$ spacing, covering $700 \mathrm{~km}^{2}$, also combined with an array of FD telescopes. Both experiments study the spectrum, the origin, and the composition of UHECR.

Recently, joint working groups have been investigating some differences between Auger and TA results, e.g. the energy at which the flux suppression occurs [3], the interpretations of that suppression, and the composition of the UHECR primaries at the highest energies [4]. From a detection standpoint, the Auger and TA SD are not equally sensitive to the different air shower components. The Auger SD is sensitive to both the muonic and electromagnetic components and responds differently to each. The TA SD only measures charged particles and sees them all equally. Since most particles close to the core are electromagnetic, this is mostly what is measured. The muon component from air showers is an indicator of the primary cosmic ray composition and can provide a clue to revealing the origin and the acceleration mechanisms of UHECR. However, the number of muons observed with the Auger SD for each shower indicates a muon deficit in the air shower Monte-Carlo simulations [5]. The deficit ratio depends on the hadron interaction model. The number of muons observed in the data is $30 \%$ (EPOS LHC) to $80 \%$ (QGSJET II-03) larger than that of the simulation assuming proton primaries at $10^{19} \mathrm{eV}$ [6]. Understanding the origin of the discrepancy between measurements and models is critical.

In order to make direct comparisons between the SD detection techniques used by Auger and TA, a two-phase joint experimental research program is followed. The first phase consists in comparing station-level responses. In late October 2014, a water Cherenkov tank (of the "Auger North" design [10]) was deployed at the TA Central Laser Facility (CLF) [7], where the TA muon detector project is also ongoing [8]. In June 2015, a second water Cherenkov detector identical to those in Auger South was deployed adjacent to the Auger North SD station. The second phase will consist of deploying six Auger North SD stations alongside existing TA SD stations to allow for event-level comparisons of relatively low-energy showers with energies in the $10^{18} \mathrm{eV}$ range. In this paper, we present the status and prospects of this joint research project, including the measurement of the first Auger North SD data that were recorded in coincidence with TA SD shower triggers.

\section{Experiment}

The Auger South SD station is a water Cherenkov tank with three photomultiplier tubes (PMTs) that are symmetrically distributed at a distance of $1.2 \mathrm{~m}$ from the center of the tank 9 . The tank is $1.5 \mathrm{~m}$ tall and has a footprint of $10 \mathrm{~m}^{2}$. It is filled to a depth of about $1.2 \mathrm{~m}$ with about 10 $\mathrm{M} \Omega$-cm resistivity water. The water is contained in a flexible, laminated liner conforming approximately to the inner tank surface. The innermost lamination consists of Tyvek ${ }^{\circledR}$. The Cherenkov light from air shower particles is diffusively reflected inside the water volume and viewed by the 


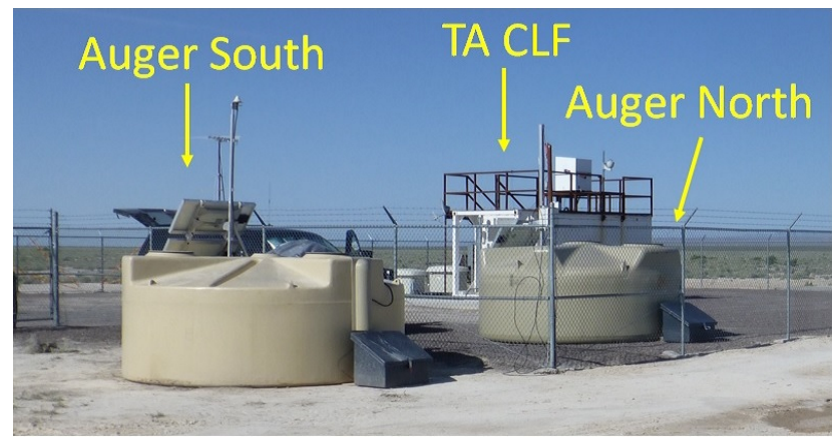

Figure 1: The two Auger SD stations deployed at the TA Central Laser Facility.

PMTs through optical windows. The signals are processed using front-end electronics having six 10-bit Fast Analog to Digital Converters (FADCs) running at $40 \mathrm{MHz}$. A dynamic range of 15 bits is realized using signals derived from the anode and from the last dynode $(\times 32)$. The digitized signals are sent to a programmable logic device board used to make various triggering decisions.

The Auger North SD station is a one PMT water Cherenkov surface detector used in the Pierre Auger Research and Development Array in Colorado, USA [11]. It is a cost-effective version of the Auger South SD station, with the same footprint, height and water volume. The Auger North and South SD stations deployed at the TA CLF are shown in figure 1 The design of the electronics for the Auger North surface detector is based on the one used at the Auger South SD. In this case however, the digitization is performed with commercial 10-bit ADCs with $100 \mathrm{MHz}$ sampling rate. The dynamic range is extended to 22 bits, using signals derived from the anode $(\times 0.1, \times 1$ and $\times 30)$ and from a deep ( $5^{\text {th }}$ out of 8$)$ dynode.

The TA SD station is composed of two layers of plastic scintillator with two PMTs, one for each layer [12]. It has an area of $3 \mathrm{~m}^{2}$ and each layer has $1.2 \mathrm{~cm}$ thickness. The scintillators and PMTs are contained in a stainless steel box which is mounted under a $1.2 \mathrm{~mm}$ thick iron roof to protect the detector from large temperature variations. Photons that are generated in the scintillator are collected by wavelength shifting fibers and read out by PMTs. The signals from PMTs are digitized by a commercial 12-bit FADC with a $50 \mathrm{MHz}$ sampling rate on the CPU board.

\section{Analysis and Results}

In order to start collecting data immediately after its deployment, the Auger North SD station was configured to record data locally. This was done by installing a large capacity (512GB) flash drive directly onto the local station controller. The second level trigger (T2) data, obtained from the standard Auger calibration procedure [1], were obtained and written on the drive at a rate of about $20 \mathrm{~Hz}$. Only a very small fraction of those events arises from UHECR showers. A smaller dataset of atmospheric muons from the T1 trigger $(100 \mathrm{~Hz})$ was also collected to derive the Vertical Equivalent Muon (VEM) calibration from the single muon energy loss spectrum. In this analysis, the data from two observation periods are used; the first is Oct. 21, 2014 - Nov. 17, 2014 and the second is Nov. 19, 2014 - Dec. 7, 2014. The flash drive was swapped between the two periods. The exchange requires a shutdown of the station to open the tank and access the local 

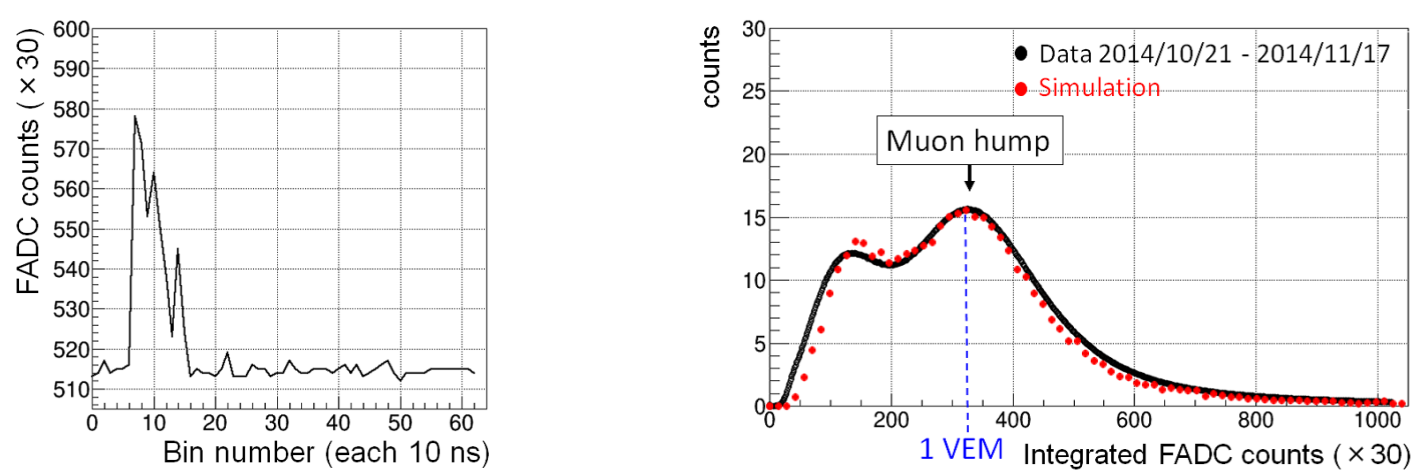

Figure 2: (left) A typical waveform of the calibration data of the Auger North SD. The anode $\times 30$ signal is used. (right) The 1 VEM histogram from the calibration data and the MC simulation. The simulation is from a CORSIKA air shower simulation in which the QGSJET II-04 hadronic interaction model and the Geant4 detector simulation are used. The peak position of the histogram for the simulation is adjusted to fit the experimental data.

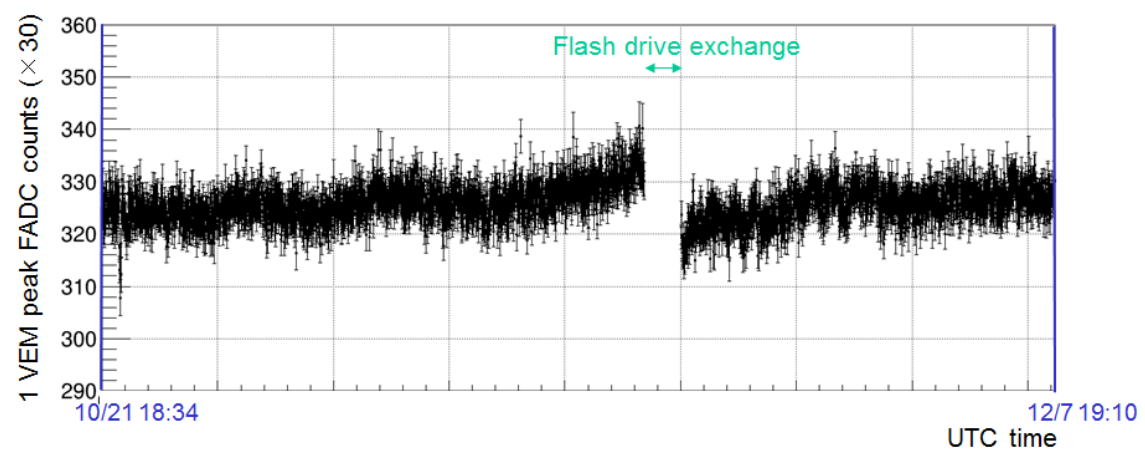

Figure 3: Time evolution of the peak position of the single muon histogram. Indicated on the figure is the time when the flash drive was swapped.

station controller. Figure2 (left) shows a typical waveform of the calibration data. Figure2(right) shows the histogram of pulse areas obtained from the FADC traces from the data and from the simulation. The air shower simulations were performed with CORSIKA in which the QGSJET II04 hadronic interaction model was used and the SD station geometry and response were simulated with GEANT4. The features of the spectrum are typical. The first peak corresponds to the residual noise suppressed by setting a threshold on the FADC integrated pulses. The second peak is the so-called "muon hump" from which the VEM can be deduced. As can be seen, the overall shape of the spectrum can be well described by the simulation. Figure 3 shows the evolution over time of the peak position of the single muon histogram. The VEM calibration appears to be relatively stable with small day/night (temperature-related) variation. An anomalous shift is observed around the time the flash drive is swapped. It is unclear at this time what caused this anomaly.

To identify actual UHECR shower events recorded by the Auger North SD station, the T2 timestamps are extracted and checked against the TA SD event trigger time within a $\pm 32 \mu \mathrm{s}$ window, corresponding to the time scale of a TA SD shower event. Air showers are reconstructed 
with the TA SD array using the method developed for the measurement of the energy spectrum by TA [16]. The air shower reconstruction program eliminates the events with poor resolution. In this analysis, we use the loose-cut selection criteria described in [17] to gain larger statistics.

Once the timestamp matching process is completed, 17 Auger North SD events are obtained in coincidence with TA SD loose-cut pass events. Among them, 10 events have core positions within $5 \mathrm{~km}$ of the Auger North SD. Figure 4 shows the display of a coincidence event. Also shown on the figure are the waveforms of the 5 TA SD and the Auger North SD stations associated with the air shower event. Based on the TA reconstruction, the energy and zenith angle of the primary cosmic ray are respectively $3.04 \times 10^{18} \mathrm{eV}$ and 46.8 degrees. Figure 5 (left) shows the waveform of the Auger North SD. The signal area is about $40 \mathrm{VEM}\left(\simeq 4 \mathrm{VEM} / \mathrm{m}^{2}\right)$ at about $1600 \mathrm{~m}$ from the shower core. The current event selection criterion for Auger-TA coincident events is that the Auger North SD trigger time is within $\pm 32 \mu$ s of the TA SD shower trigger time, but this condition possibly includes SDs triggered by background muons. Figure 5 (right) shows the corresponding distribution of the station trigger time as function of the distance between the stations and the shower core along the shower axis projected onto the ground. The Auger SD appears as the earliest trigger time among the SDs. The feature is consistent with the reconstructed shower geometry shown in figure 4 The other events present similar features.

Since March 2015, a third period of observation is ongoing, hence more results may be shown by the time of the conference. In June 2015, the second Auger SD station was deployed in the field. Both Auger SD stations at the CLF are now connected to a single board computer (SBC), which can be accessed remotely. In the near future, a TA SD station will be installed at the CLF and a local trigger will be formed between the Auger and TA SD stations, including the larger muon detector also installed at the CLF. Both the local and TA shower triggers will be provided to the Auger SBC to request data collection from the Auger SD stations. In this way, only data from higher level triggers will be collected. By combining the information of the three detector types, one will be able to thoroughly compare the response of the individual Auger and TA SD stations and estimate the relative contributions of the muon and EM components at the CLF location for showers triggered by TA.

The second phase of this joint collaboration aiming at making event-level comparisons will require the deployment of six contiguous Auger North SD stations alongside existing TA SD stations. The site for such a micro-Auger array has been identified and the deployment will occur towards the end of the year. The current plan is to have the Auger SD micro-array trigger independently to compare trigger efficiencies and energy estimators. Information from the Auger and TA lateral distribution functions should also provide insights on the muon and EM components of the observed showers.

\section{Summary}

A joint Auger-TA experimental research program studying the difference in SD responses is ongoing. Initial results are promising. The Auger North SD station VEM calibration appears to be well understood by the simulation, and first Auger-TA SD coincidences have been observed by matching the Auger SD station timestamps to the TA shower trigger in post processing. In June 


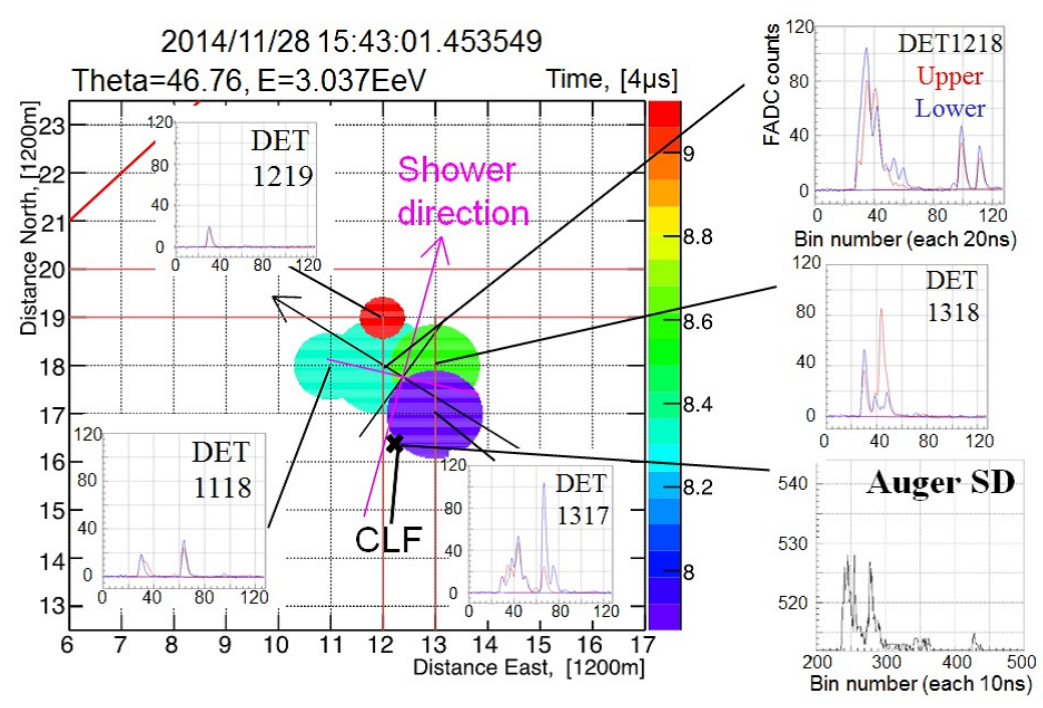

Figure 4: The event display for the event discussed in the text. The circle centers correspond to the TA SD positions. The circle areas are proportional to the logarithm of the TA SD station pulse heights. The circle colors represents the TA SD detection times from the detection time of the first triggered detector within the same $\pm 32 \mu \mathrm{s}$. The magenta arrow shows the shower axis projected onto the ground and the black $\mathrm{x}$ mark is the position of the Auger North SD at the CLF. The red line of the TA SD waveform is the upper layer signal, while the blue line shows the lower layer signal.

2015, a second Auger (South) station was deployed in the field, and both Auger SD stations were connected to a SBC inside the CLF. This opens the door to the direct collection of higher-level local and global triggers allowing for more robust studies. The joint collaboration is also actively preparing the development of a micro-Auger array within TA for event-level comparative studies.

\section{References}

[1] A. Aab et al. (Pierre Auger Collaboration), The Pierre Auger Cosmic Ray Observatory, accepted for publication in Nucl. Instrum. Meth. A (2015) arXiv: 1502.01323.

[2] P. Tinyakov et al. (Telescope Array Collaboration), Latest results from the telescope array, Nucl. Instrum. Meth. A742 (2014) 29 - 34.

[3] A. Aab et al., (Pierre Auger collaboration), Measurement of the cosmic ray spectrum above $4 \times 10^{18}$ eV using inclined events detected with the Pierre Auger Observatory, submitted to JCAP (2015) arXiv: 1503.07786.

[4] R. Abbasi et al., for the Pierre Auger Collaboration and the Telescope Array Collaboration, Report of the Working Group on the Composition of Ultra High Energy Cosmic Rays, to appear in the Proceedings of the UHECR workshop, Springdale, USA (2014) arXiv:1503.07540.

[5] A. Yushkov for the Pierre Auger Collaboration, Measurement of the muon shower content at the Pierre Auger Observatory, EPJ. Conf. 53, 07002 (2013). 

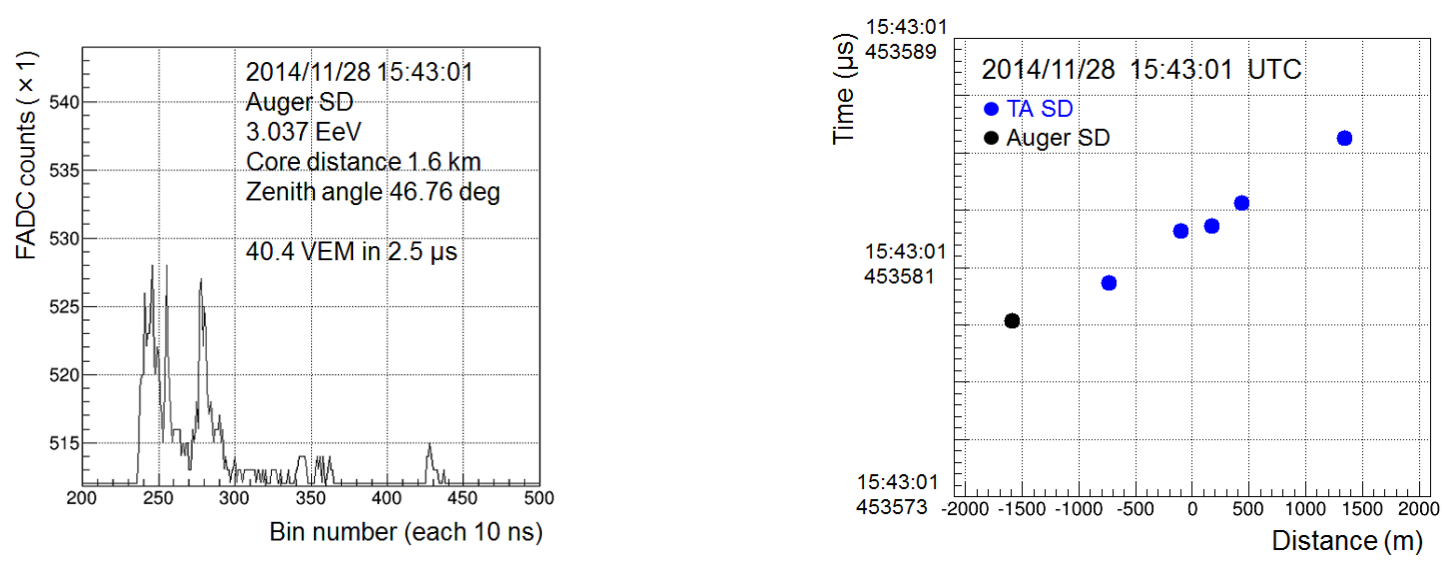

Figure 5: (left) The waveform of the Auger North SD station for the event discussed in the text. (right) The distribution of the station trigger time as function of the distance between the stations and the shower core along the shower axis projected onto the ground.

[6] A. Aab et al., (The Pierre Auger Collaboration), Muons in air showers at the Pierre Auger Observatory: Mean number in highly inclined events, Phys. Rev. D. 91, 032003 (2015) arXiv:1408.1421v5.

[7] S. Udo et al., The Central Laser Facility at the Telescope Array, Proc. 30th ICRC 5, 1021 (2007) Merida, Mexico.

[8] T. Nonaka et al., Performance and status of TA muon detector, these proceedings.

[9] I. Allekotte et al., The surface detector system of the Pierre Auger Observatory, Nucl. Instrum. Meth. A586 (2008) 409 - 420, arXiv: 0712.2832.

[10] J. Blümer et al. (The Pierre Auger Collaboration), The northern site of the Pierre Auger Observatory, New Journal of Physics, 12, 035001 (2010).

[11] F. Sarazin et al., The Pierre Auger Research and Development Array (RDA) in southeastern Colorado - R\&D for a giant ground array, EPJ. Conf. 53, 08017 (2013).

[12] T. Abu-Zayyad et al., The surface detector array of the Telescope Array experiment, Nucl.Instrum. Meth. A689 (2012) 87 - 97.

[13] M. Teshima et al., Properties of $10^{9}-10^{10} \mathrm{GeV}$ extensive air showers at core distances between 100 and 3000 m, J. Phys. G: Nucl. Phys. 12 (1986) 1097 - 1113.

[14] M. Takeda et al., Extension of the Cosmic-Ray Energy Spectrum beyond the Predicted Greisen-Zatsepin-Kuz'min Cutoff, Phys. Rev. Lett. 81 (1998) 1163 - 1166.

[15] M. Takeda et al., Energy determination in the Akeno Giant Air Shower Array experiment, Astropart. Phys. 19 (2003) 447 - 662.

[16] T. Abu-Zayyad et al., The Cosmic Ray Energy Spectrum Observed with the Surface Detector of the Telescope Array Experiment, ApJ, 768, L1 (2013).

[17] R. U. Abbasi et al., Indications of Intermediate-Scale Anisotropy of Cosmic Rays with Energy Greater than $57 \mathrm{EeV}$ in the Northern Sky Measured with the Surface Detector of the Telescope Array Experiment, ApJ, 790, L21 (2014). 